\title{
NONDESTRUCTIVE EXAMINATION OF FUEL CHANNELS IN PHWR NUCLEAR POWER PLANTS
}

\author{
A. Savin ${ }^{1}$, L. Udpa ${ }^{2}$, R. Steigmann ${ }^{1}$, R. Grimberg ${ }^{1}$, S. Udpa ${ }^{2}$ \\ ${ }^{1}$ Nondestructive Testing Department \\ National Institute of Research and Development for Technical Physics \\ 47 D. Mangeron Blvd., 700050, Iasi, Romania \\ ${ }^{2}$ Department of Electrical and Computer Engineering \\ Michigan State University \\ Engineering Building, \\ East Lansing, MI 48824 - 1226, USA
}

\begin{abstract}
This paper proposes the use of a new type of eddy current transducer - the inner eddy current transducer with rotating magnetic field that it was experimented to detect the discontinuities in un-irradiated pressure tubes, as well as to determine the position and tilt of garter springs. This transducer was used to determine the hydrides present zones and of delayed hydride cracking, the zones in which $\mathrm{ZrO}_{2}$ is present and the thickness of oxide layers.
\end{abstract}

Keywords: Tube inspection, Nuclear power plants, Eddy current examination, Zirconium, Hydride, Oxide

\section{Introduction}

A critical part of pressurized heavy water reactor (PHWR) CANDU 600MW(e) type Nuclear Power Plant is the calandria vessel (Fig. 1) made of austenitic steel, two integral end shield (also made of austenitic stainless steels) with carbon steel shielding balls each. Horizontally penetrated by 380 lattice tubes, 380 Zircaloy - 2.5 calandria tubes, joining the lattice tubes at each position in lattice and 380 fuel channels assemblies mounted within those lattice sites.

The fuel channel assembly is presented in Fig. 2 [1]:

To inspect fuel channel, using eddy current techniques, in literature the use of bobbin coil [2], segmented coils [3] and $\mu^{+}$probe [4] are indicated.

In this paper we propose the use of a new type of eddy current transducer - the inner eddy current transducer with rotating magnetic field that it was experimented to detect the discontinuities in un-irradiated pressure tubes [5], as well as to determine the position and tilt of garter springs [6]. This transducer was used to determine the hydrides present zones and of delayed hydride cracking, the zones in which $\mathrm{ZrO}_{2}$ is present and the thickness of oxide layers. 


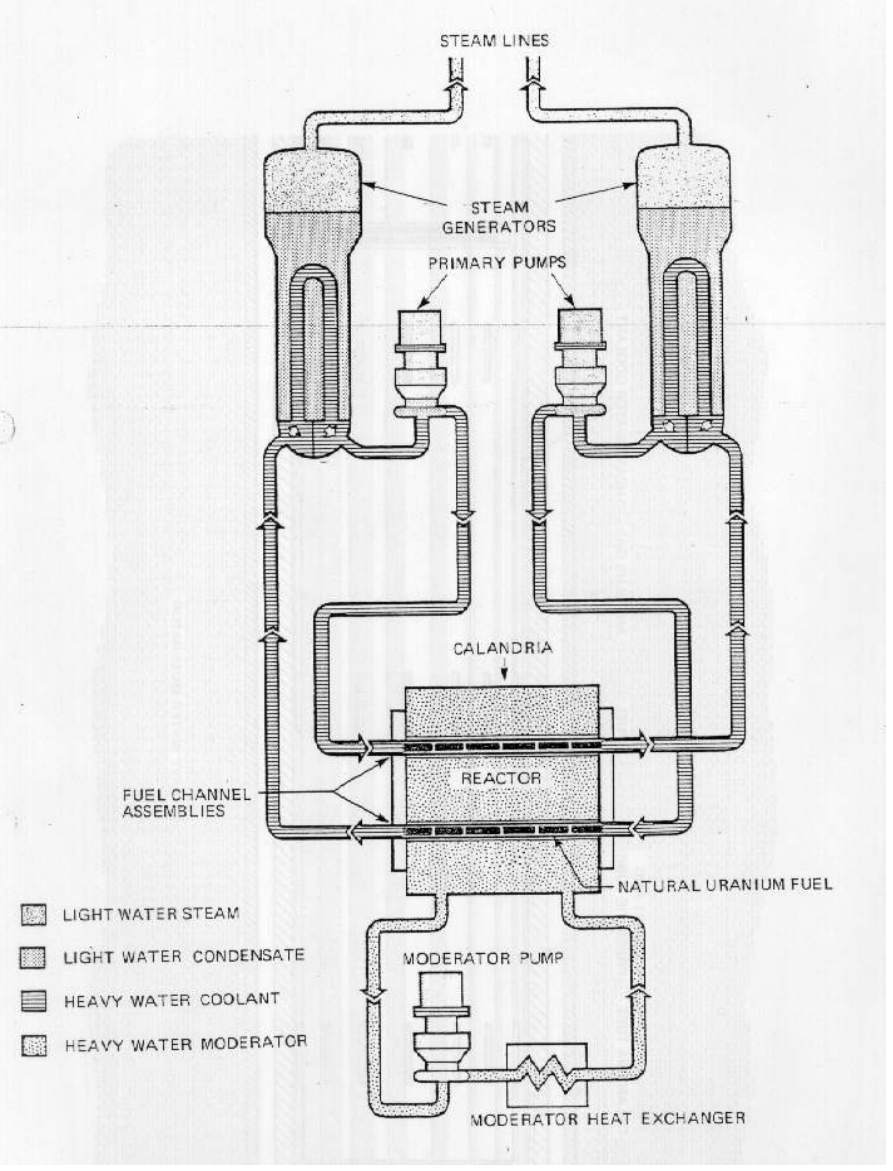

Fig. 1: Basic scheme of reactor.

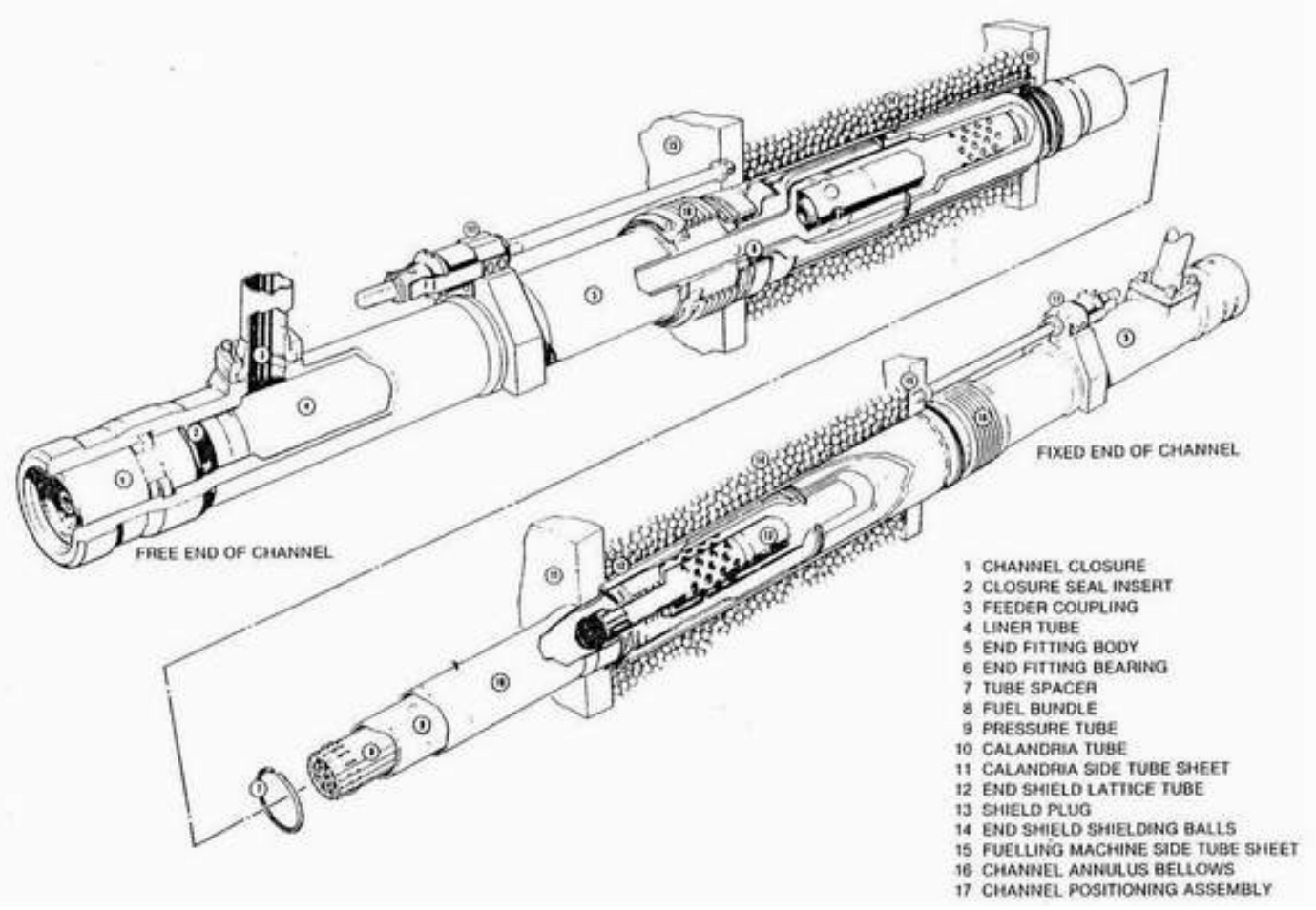

Fig. 2: Fuel channel assembly. 


\section{Zr-2.5\% $\mathrm{Nb}$ alloy and appearance of blisters and delayed hydride cracking}

$\mathrm{Zr}-2.5 \% \mathrm{Nb}$ alloy used to made pressure tubes by extrusion, has the chemical composition: $\mathrm{Nb}$ $2.4 \div 2.8$ ut\%, Oxygen 900 $\div 1300$ pm, Zirconium - balance [5].

The classical oxidation reaction of $\mathrm{Zr}$ alloy is

$$
\mathrm{Zr}+2 \mathrm{H}_{2} \mathrm{O}\left(\text { or } \quad 2 \mathrm{D}_{2} \mathrm{O}\right) \rightarrow \mathrm{ZrO}_{2}+2 \mathrm{H}_{2}\left(2 \mathrm{D}_{2}\right) \uparrow
$$

The reaction is favored by the temperature of $\mathrm{D}_{2} \mathrm{O}$ used as coolant agent $\left(256 \div 310^{0} \mathrm{C}\right)$, by the pressure of the coolant agent $(\sim 10 \mathrm{MPa})$ and by the prolonged exposure of material to internal neutrons fluxes and $\gamma$ rays [6].

In Fig. 3 is presented the evolution of $\mathrm{ZrO}_{2}$ layer thickness function of the operating time in the reactor [6]

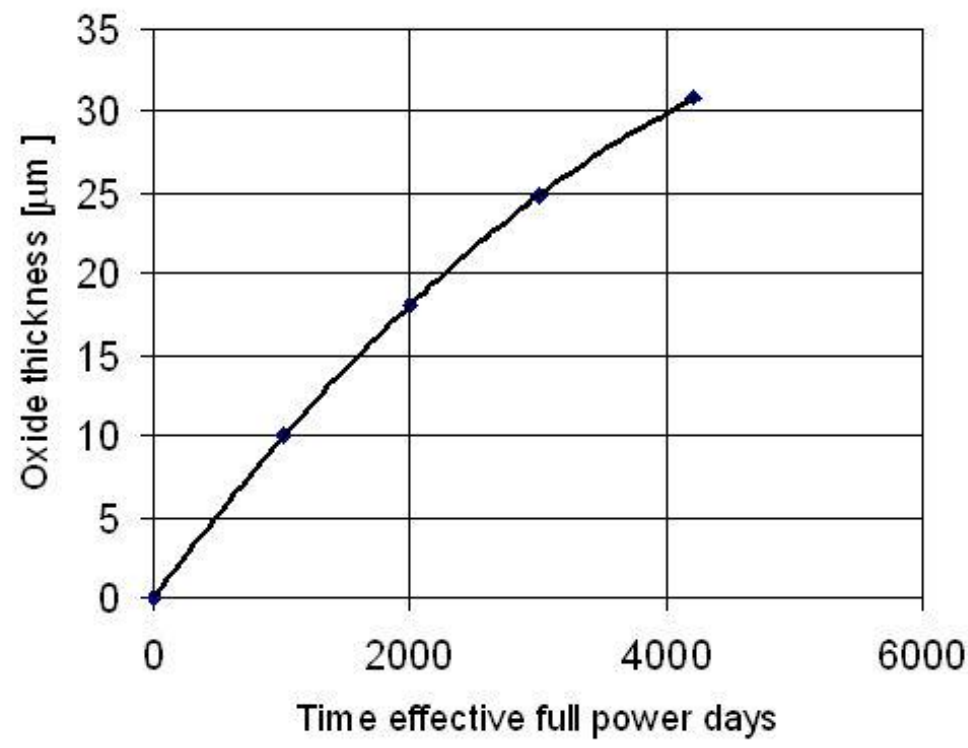

Fig. 3: $\mathrm{ZrO}_{2}$ layer thickness vs. operating time.

For the most severe loss-of -coolant accident (LOCA) in CANDU Reactors it is predicted that decay heat generated in some of fuel channels is initially transferred to the cool heavy water moderator surrounding the fuel channel. A key factor in determining the rate of the heat transfer is the magnitude of thermal contact conductance or resistance to heat flow at the interface between the pressure and calandria tubes, since it is generally known that $\mathrm{ZrO}_{2}$ is a poor thermal conductor, and much less ductile than the parent metal, its presence on the pressure tube surface would lead to a higher resistance to heat flow.

This is the reason for which the oxide layer thickness must be nondestructive determined, without direct access.

Oxidation of the Zirconium alloy surface in the presence of water results in the evolution of Hydrogen (Deuterium). The hydrogen released is very soluble in the $\mathrm{Zr}$ metal matrix where it tends to accumulate. The range of 20 to $60 \mu \mathrm{g} / \mathrm{g}$ of $\mathrm{Zr}$ results in the precipitation of Zirconium hydride $\left(\mathrm{ZrH}_{2}\right)$ (Fig. 4). The presence of $\mathrm{ZrH}_{2}$ combined with pressure tube to calandria tube contact lead to the formation of hydride blisters and the eventual cracking of the pressure tube material as the result of the stresses from blister growth.

From this reason, with the occasion of nondestructive testing that are effecting on fuel channel, the zones with hydride presence and delayed hydrogen cracking must be determined. 


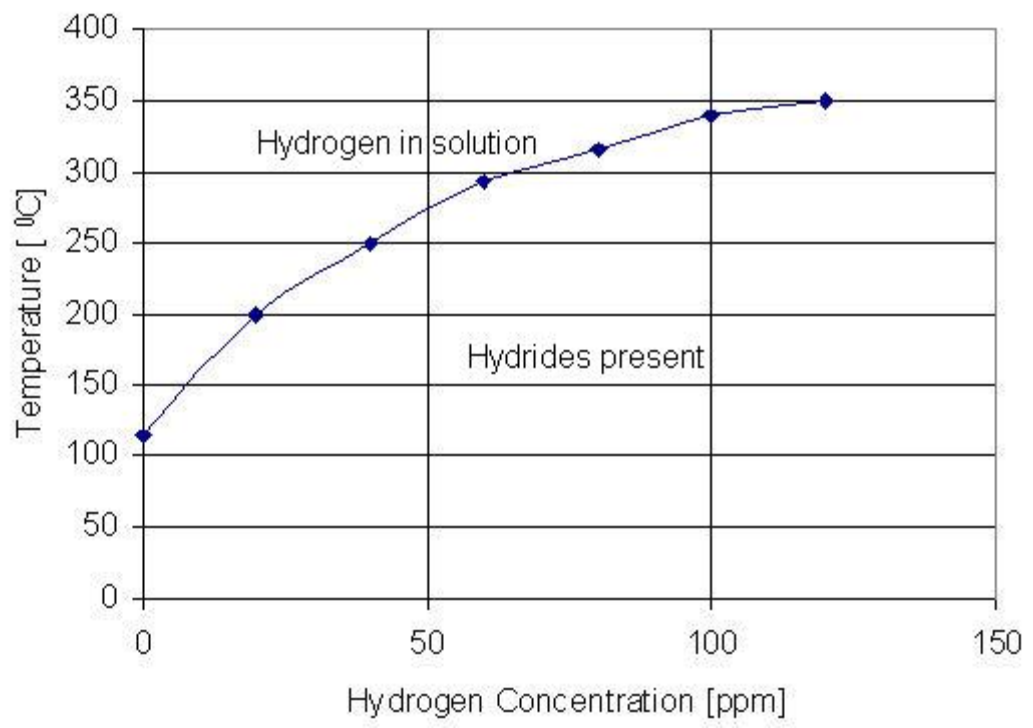

Fig. 4: Effect of temperature on the Solubility of Hydrogen in Zr-2.5\%Nb.

\section{Inner eddy current transducer with rotating magnetic field}

The transducer is absolute send-receiver type, and a schematic of the transducer is presented in Fig. 5. The transmitter is made from 3 rectangular coils, wound with $2 \pi / 3$ angle between them, star connected and supplied with a three-phase electric current. The vector addition of the magnetic fields created by the 3 coils results in a magnetic rotating field, with the frequency being equal to that of the three-phase current. The receiver is made of an equally spaced array 24 identical coils, which are consecutively interrogated through an analogical multiplexer.

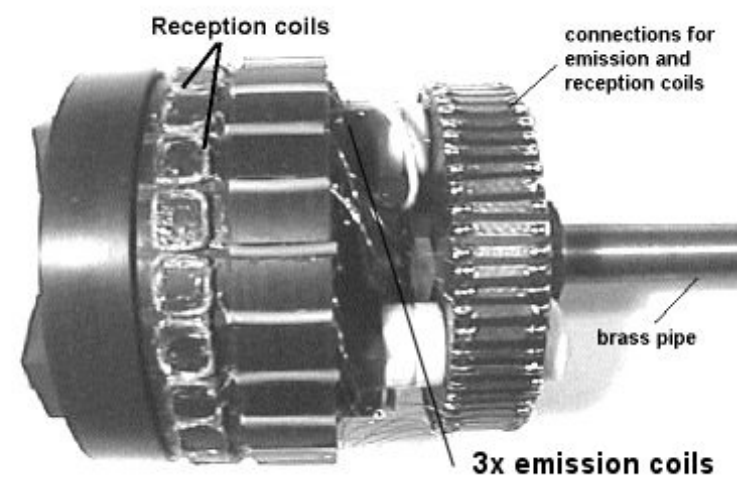

a)

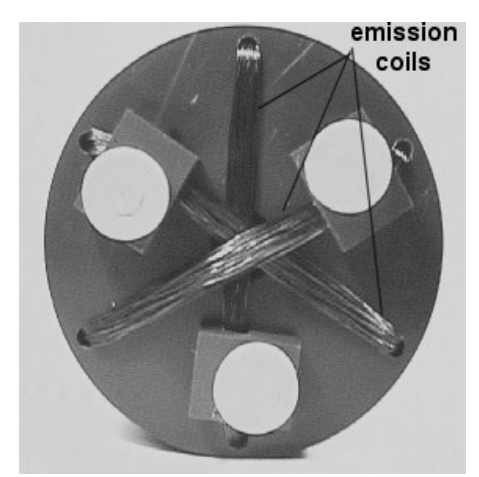

b)

Fig. 5: The inner eddy current transducer with rotating magnetic field.

a) general view; b) front view.

\section{Experimental set-up}

The experimental set-up is presented in Fig. 6. Three programmable function generator cards AWG 7223 PC-2 type supply the circuit with the three phase alternative current, as well as the reference for synchronous detection. The signals are amplified and applied to the transmitter creating a rotating magnetic field. The 24 receiver coils are consecutively interrogated, and connected to the input of an SFT 6000N eddy current board. 


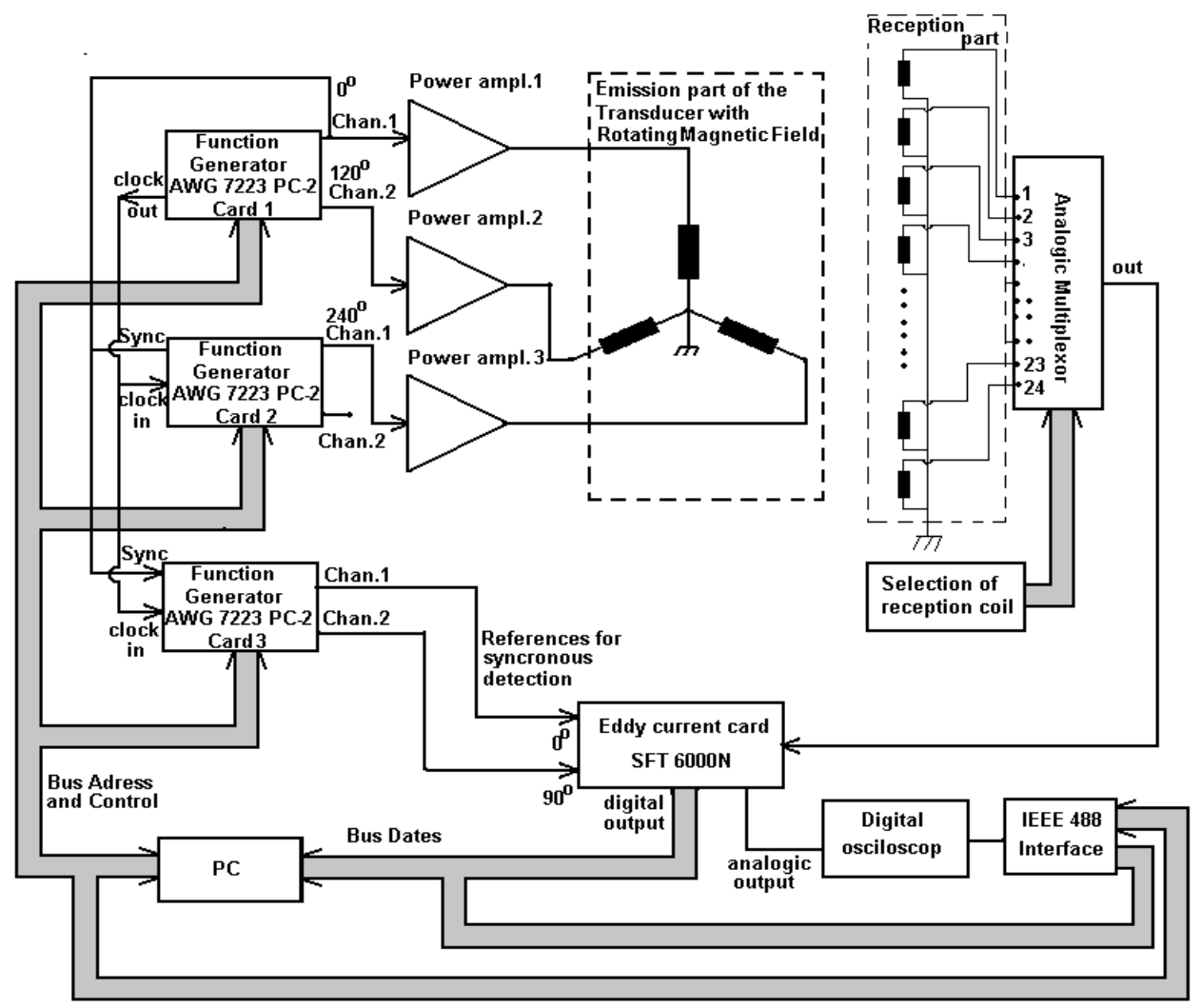

Fig. 6: Experimental set-up.

\section{5. $\mathrm{ZrO}_{2}$ layer thickness determination and evaluation of hydride blisters}

The electric conductivity of $\mathrm{Zr}-2.5 \%$ Nbis $1.89 * 10^{6} \mathrm{~S} / \mathrm{m}$. $\mathrm{ZrO}_{2}$ has properties of electric insulator at usage temperature of coolant agent and moderator. Due to the fact that the oxide layer isn't continuum, the electric conductivity of the layer can be considered having the value $3900 \mathrm{~S} / \mathrm{m}$. The Zirconium hydrides that precipitates and finally forming blisters, has the conductivity on the range of $1.1 * 10^{6} \mathrm{~S} / \mathrm{m}$.

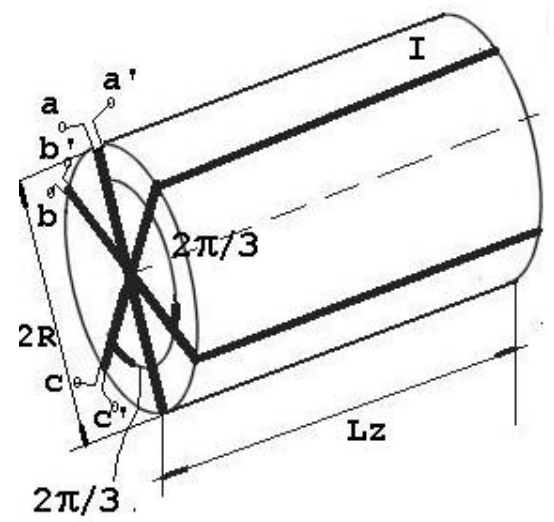

a

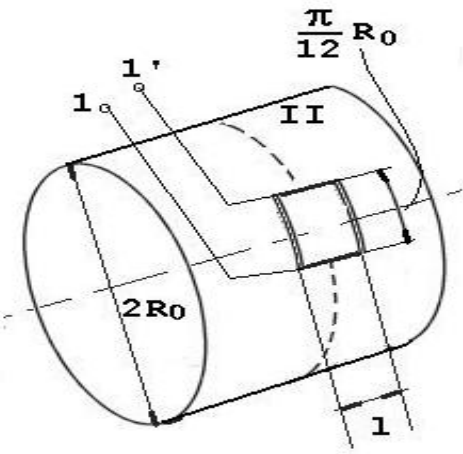

b

Fig. 7: Inner eddy current transducer with rotating magnetic field:

a - emission part,

$\mathrm{b}$ - reception part. 
To determine the layer thickness of $\mathrm{ZrH}_{2}$ and $\mathrm{ZrO}_{2}$, the eddy current methods with bobbin coil [9] or pancake transducers [10] are used. The using of eddy current transducer with rotating magnetic field has proved to be fruitful to emphasize the discontinuities in pressure tubes and to locate the position and the tilt of garter springs. Therefore we proposed to extend its employment to determine the $\mathrm{Zr}$ oxide and hydride layer thickness.

The emission part of the transducer with rotating magnetic field, it means the source that generates the field created by transducer is schematically presented in Fig. 7(a).

In cylindrical coordinate $(\rho, \varphi, z)$, the component of current source can be write

$$
\begin{aligned}
& J_{\rho}=I_{0}\left[\delta\left(z-\frac{L_{z}}{2}\right)-\delta\left(z+\frac{L_{z}}{2}\right)\right] \\
& *\left[\delta(\Phi)-\delta(\Phi-\pi)+e^{j \frac{2 \pi}{3}}\left(\delta\left(\Phi-\frac{2 \pi}{3}\right)-\delta\left(\Phi-\frac{5 \pi}{3}\right)\right)+e^{j \frac{4 \pi}{3}}\left(\delta\left(\Phi-\frac{\pi}{3}\right)-\delta\left(\Phi-\frac{4 \pi}{3}\right)\right)\right] \\
& * \int_{0}^{R} \delta\left(\rho-\rho_{0}\right) d \rho_{0} \\
& J_{\Phi}=0 \\
& J_{z}=I_{0} \delta(\rho-R) \\
& *\left[\delta(\Phi)-\delta(\Phi-\pi)+e^{j \frac{2 \pi}{3}}\left(\delta\left(\Phi-\frac{2 \pi}{3}\right)-\delta\left(\Phi-\frac{5 \pi}{3}\right)\right)+e^{j \frac{4 \pi}{3}}\left(\delta\left(\Phi-\frac{\pi}{3}\right)-\delta\left(\Phi-\frac{4 \pi}{3}\right)\right)\right] \\
& * \int_{L_{z}}^{\frac{l_{z}}{2}} \delta\left(z-z_{0}\right) d z_{0} \\
& \frac{L_{2}}{2}
\end{aligned}
$$

where $\delta$ is Dirac functional and and $\mathrm{L}_{\mathrm{z}}$ is the length of one of three rectangular coils that made the emission. The field created by source in the pressure tube's wall can be writing using the dyadic Green's functions method [11]

$$
\bar{E}_{2}(\bar{r})=j \omega \mu_{0} \int_{\text {Vsource }} \vec{G}_{12}\left(\bar{r}, \bar{r}^{\prime}\right) \bar{J}\left(\bar{r}^{\prime}\right) d \bar{r}^{\prime}
$$

where $\omega$ is angular frequency of the tri-phased current through source, $\bar{J}\left(\bar{r}^{\prime}\right)$ has the components given in (1) and $\vec{G}_{12}\left(\bar{r}, \bar{r}^{\prime}\right)$ is dyadic Green's function air-material with the expression given in [11]

$$
\begin{aligned}
& \vec{G}_{12}=\frac{j}{8 \pi} \sum_{m=-\infty}^{\infty} \int_{-\infty}^{\infty} d k_{z} \frac{1}{\left(k_{1} k_{2 \rho}\right)^{2}} \vec{D}_{\mu}\left[H_{n}^{(1)}\left(k_{2 \rho} \rho\right) \vec{I}+J_{n}\left(k_{2 \rho} \rho\right) \bar{R}_{23}\right] \\
& * \widetilde{M}_{2} * \widetilde{T}_{12} * \widetilde{M}_{1} * J_{n}\left(k_{1 \rho} \rho^{\prime}\right) \vec{I} e^{j\left[n\left(\Phi-\Phi^{\prime}\right)+k_{z}\left(z-z^{\prime}\right)\right]} \overleftarrow{D}_{\varepsilon}{ }^{\prime t}
\end{aligned}
$$

Because the electric conductivity of oxidation zones and those in which zirconium hydride precipitation exist, is different by the conductivity of host material, these regions will behave like auxiliary current sources with density

$$
\bar{J}_{r}(\bar{r})=\left[\sigma_{r}(\bar{r})-\sigma\right] \bar{E}_{d}(\bar{r})
$$


where $\sigma_{r}(\bar{r})$ is the conductivity of the oxidation zone or hydride accumulation, $\sigma$ is the electric conductivity of $\mathrm{Zr}-2.5 \% \mathrm{Nb}$ alloy and $\bar{E}_{d}(\bar{r})$ is the electric field scattered by these regions.

The electric field in the tube's wall can be write

$$
\bar{E}_{2}(\bar{r})=\bar{E}_{d}(\bar{r})-j \omega \mu_{0} \sigma \int_{V r} \vec{G}_{22}\left(\bar{r}, \bar{r}^{\prime}\right) \frac{1}{\sigma} \bar{J}_{r}\left(\bar{r}^{\prime}\right) d \bar{r}^{\prime}
$$

with $\mathrm{V}_{\mathrm{r}}$ the volume occupied by oxide or hydride layer and $\vec{G}_{22}(\bar{r}, \bar{r})$ is dyadic Green's function for material-material, of whom expression is given in [11]

$$
\vec{G}_{22}\left(\bar{r}, \bar{r}^{\prime}\right)=\frac{j}{8 \pi^{2}} \sum_{n=-\infty}^{\infty} \int_{-\infty}^{\infty} d k_{z} \frac{1}{\left(k_{2} k_{2 \rho}\right)^{2}} \bar{D}_{\mu} \bar{F}_{n}\left(\rho, \rho^{\prime}\right) e^{j n\left(\Phi-\Phi^{\prime}\right)+k_{z}\left(z-z^{\prime}\right)} \frac{\overleftarrow{D_{\varepsilon}}}{2}+\frac{\hat{\rho} \hat{\rho}}{k_{2}{ }^{2}} \delta\left(\bar{r}-\bar{r}^{\prime}\right)
$$

This equation is Fredholm integral equation of the first kind having as unknown the field scattered on material inhomogeneities as oxidation zones or where Zirconium hydride accumulations exists, $\bar{E}_{d}(\bar{r})$.

At the reception part of the eddy current transducer with rotating magnetic field (in Fig. 7b), the electromotive force in one of reception coils will be

$$
e=\iint_{\Psi} \bar{E}_{1} d \bar{\ell}
$$

where $\psi$ is the outline of one of reception coil and $\bar{E}_{1}(\bar{r})$ is given by

$$
\bar{E}_{1}(\bar{r})=\int_{V r} \vec{G}_{21}\left(\bar{r}, \bar{r}^{\prime}\right) \bar{J}_{e}\left(\bar{r}^{\prime}\right) d \bar{r}^{\prime}
$$

with $\vec{G}_{21}\left(\bar{r}, \bar{r}^{\prime}\right)$ dyadic Green's function material -air and $\bar{J}\left(\bar{r}^{\prime}\right)$ given by (4).

The dyadic Green's function $\vec{G}_{21}\left(\bar{r}, \bar{r}^{\prime}\right)$ has the expression

$$
\begin{aligned}
& \vec{G}_{21}\left(\bar{r}, \bar{r}^{\prime}\right)=\frac{j}{8 \pi^{2}} \sum_{n=-\infty}^{\infty} \int_{-\infty}^{\infty} \frac{1}{\left(k_{2} k_{2 \rho}\right)^{2}} \bar{D}_{\mu}\left[J_{n}\left(k_{1 \rho} \rho\right) \bar{I}\right] * \widetilde{\bar{M}}_{1} * \widetilde{\bar{T}}_{21} * \widetilde{\bar{M}}_{2} \\
& {\left[H_{n}^{(1)}\left(k_{2 \rho} \rho^{\prime}\right) \bar{I}+J_{n}\left(k_{2 \rho} \rho^{\prime}\right) \widetilde{\bar{R}}_{23}\right] e^{j n\left(\Phi-\Phi^{\prime}\right)+j k_{z}\left(z-z^{\prime}\right)} \overline{\bar{D}}_{\varepsilon}{ }^{t}}
\end{aligned}
$$

The integral equation (9) hasn't exact solution [12] and therefore must be numerically solved, one method being the moments method [13]. The moments method consist in the application of a uniform mesh in the region in which the inhomogeneity exists, supposing that the electric field in any of mesh cells is constant, equal with the field in the centre of cell. In the case in which a region of inhomogeneity occupies exactly a part of cell, the weight of cell is 1 ; in the case in which a region of inhomogeneity occupies just a part of cell, the weight will be the ratio between the volume of inhomogeneity and of elementary cell.

From here results that the operation of the eddy current control can be write as matrix form

$$
e_{i}=A_{i j} \sigma_{r_{j}}+\varepsilon_{i} \quad i=1,2, \ldots N_{s} ; j=1,2, \ldots N_{c}
$$


with $\mathrm{N}_{\mathrm{s}}$ represents the points in which emf of one reception coil is measured, $\mathrm{N}_{c}$ is number of cells, $e_{i}$ is the emf measured in the point $i, \sigma_{r j}$ is the conductivity of $j^{\text {th }}$ cell of the mesh, $A_{i j}$ represents the matrix of the model obtained using the above equations, $\varepsilon_{\mathrm{i}}$ is the totality of measure noises, variable lift-off, the imperfections of the model and numerical calculation errors. Neglecting the noises, the solution of eq. (10) can be write

$$
\sigma_{r}=\overline{\bar{A}}^{t} \bar{e}
$$

where

$$
\overline{\bar{A}}^{t}=(\overline{\bar{A}} A)^{-1} \overline{\bar{A}}^{T}
$$

is the inverse in Moore-Penrose sense of the matrix $\overline{\bar{A}}$, the superior index $\mathrm{T}$ marking the matrix's transpose and -1 index representing the inverse matrix.

\section{Experimental results}

On non-irradiated pressure tubes samples made from $\mathrm{Zr}-2.5 \% \mathrm{Nb}$ alloy, having $03 \mathrm{~mm}$ inner diameter and $4.2 \mathrm{~mm}$ wall thickness, approximate circularly oxide zones with average diameter $10 \mathrm{~mm}$ and $\sim 40 \mu \mathrm{m}$ thickness of oxide layer.

In Fig. 8 is presented the dependency of real and imaginary components of induced signal into one of reception coil of the inner transducer with rotating magnetic field that pass over the oxidation zone by position of the middle of the transducer.

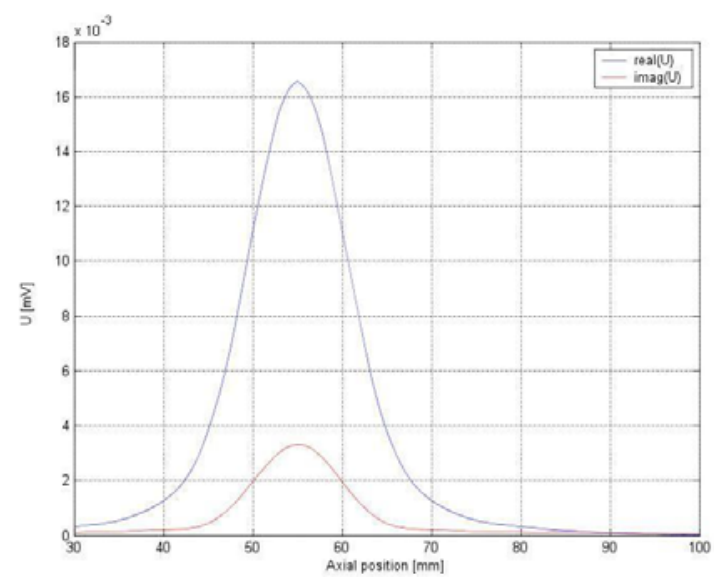

Fig. 8: Dependence of signal due to oxide zone by the position of transducer.

The tests were made in the frame of round robin blind test.

It is observed that the presences of oxide layers with typically thickness that can appear during lifetime of reactor are clearly emphasized.

Through specific techniques, on the same samples, hydride zirconium precipitate that has evolutes until the apparition of blisters has been made. The response of inner eddy current transducer with rotating magnetic field at the blister presence is given in Fig. 9. The blister has $0.3 \mathrm{~mm}$ average diameter and $0.1 \mathrm{~mm}$ depth. 


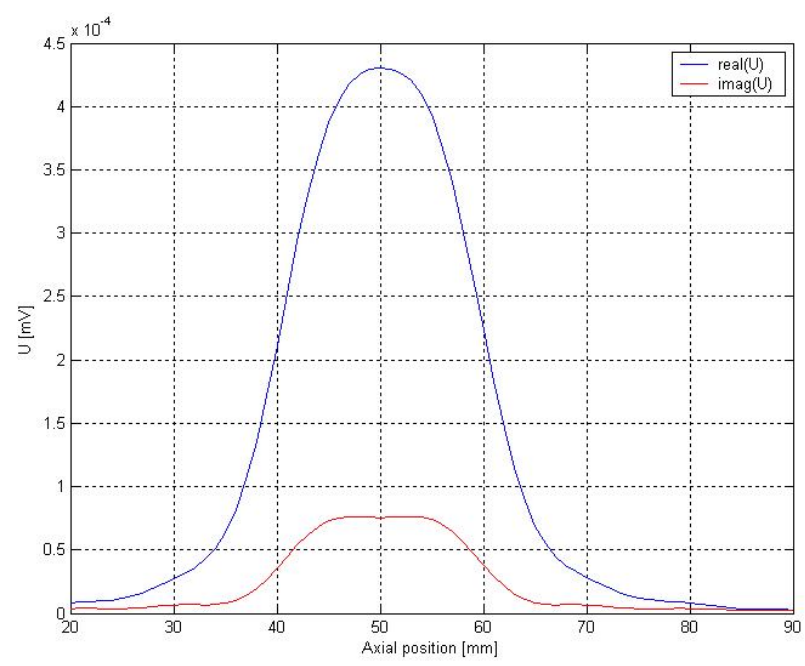

Fig. 9: Dependence of signal due to presence of blister by the position of transducer.

\section{Conclusions}

The eddy current inner transducer with rotating magnetic field allows the determination with enough precision of the dimensions and positions of oxidation zones and blisters that can appear on pressure tubes during the functioning of reactor.

In a previous paper we shown the capability of this transducer to localize and determination of garter springs position and tilt and specific discontinuities that can appear at pressure tubes. This fact recommends the described transducer and numerical signal processing methods as adequate for the testing of pressure tubes during the outage.

\section{Acknowledgment}

This paper is partially supported by NSF USA under Grant \#0303914 and IAEA Vienna under Contract No.11164R4.

\section{References}

[1] CANDU Nuclear Power System, Report AECL -TDS1-105, 1981.

[2] Report of IAEA Consultancy on Technology Advances in Pressure Tubes Maintenance and Replacement, IAEA-CT-3111, 1996.

[3] Bhole W.M., Tripothi U.N., Kumar A., Kulkarni P.G., INSIGHT, (38), 1996, 718-721

[4] Report of the Coordinated Research Project on Intercomparison of Techniques for Pressure Tube Inspection and Diagnostics - CRP 13.30.10 IAEA -622-13-03-CT10213, Vienna 2003.

[5] Grimberg R, Udpa L., Savin A., Steigmann R., Udpa S.S, Inner Eddy Current Transducer with Rotating Magnetic Field; Experimental Results: Application to Nondestructive Examination of Pressure Tubes in PHWR Nuclear Power Plants, Research in Nondestructive Evaluation, Springer-Verlag New York, LLC, Volume 16, Issue 2, 2005.

[6] Science and Reactor Fundamentals Materials; CNSC Technical Training Group, Rev.1 Jan.2003, AECL-97146.

[7] Elmoselhi M.B., Warr B.D., Intyre S.Mc, Zirconium in Nuclear Industry, $10^{\text {th }}$ International Symposium ASTM, STP1245, 1994,62-72.

[8] Corrosion of Zirconium Alloys in Nuclear Power Plants, IAEA-TECDOC-996, 1998. 
[9] Alois A., Mendoca H., Ruch M., Eddy Current Assessment of Hydrogen content in Zirconium Based Alloys, Proceedings of $15^{\text {th }}$ World Conference on Nondestructive Testing, Rome, Italy, 2000, IDN 332.

[10] Prémel D, Savin A, Grimberg R, Madaoui N., Modelling the operations of a new type of eddy current transducer for non-destructive evaluation of tubes of steam generators, Studies in Applied Electromagnetics and Mechanics 17 Electromagnetic Nondestructive Evaluation (IV) S.S. Udpa et al.(Eds) IOS Press, (2000),42-49.

[11] Grimberg R, Udpa L., Savin A., Steigmann R., Udpa S.S, Inner Eddy Current Transducer with Rotating Magnetic Field: Theoretical Model - Forward Problem, Research in Nondestructive Evaluation, Springer-Verlag New York, LLC, Volume 16, Issue 2, 2005.

[12] Morse P.M., and Feshbach H., (1953) Methods of theoretical physics: McGraw-Hill, Inc.

[13] Harrington R.F., Field Computation by Moment Method, edII, Wiley, IEEE Press, N.Y.1999. 